\title{
Amplifier and laser demonstrations in Bi-doped silica optical fibers
}

\author{
J. K. Sahu, N. K. Thipparapu, A. A. Umnikov, P. Barua and M. Nunez Velazquez \\ Optoelectronics Research Centre, University of Southampton, Highfield, Southampton, SO17 1BJ, U.K. \\ Authore-mail address: jks@orc.soton.ac.uk
}

\begin{abstract}
We will review the influence of fiber fabrication and selection of pump wavelengths on gain, noise figure and laser efficiency of Bismuth (Bi)-doped fiber amplifiers and lasers operating in the wavelength region of $1150-1400 \mathrm{~nm}$.

OCIS codes: (060.2320) Fiber optics amplifiers and oscillators; (060.2270) Fiber characterization; (060.2280) Fiber

design and fabrication; (060.2290) Fiber materials.
\end{abstract}

\section{Introduction}

Over the years the performance of rare earth (RE)-doped fibers have been improved significantly in the wavelength bands around 1, 1.5 and $2 \mu \mathrm{m}$ using Ytterbium, Erbium and Thulium or Holmium, respectively [1]. Concurrently, exploring new dopant materials as a gain media in optical fibers has become a burgeoning research area [2, 3], especially for the wavelength bands inaccessible by RE-elements. The interest stems from the widespread applications offered by the amplifiers and lasers in these wavelength bands such as medicine, astronomy, material processing and particularly for extended optical fiber communications [3]. Bismuth (Bi)-doped fibers in different glass hosts (i.e., aluminoslicate, phosphosilicate and germanosilicate) have shown broad luminescence covering from 1150-1800nm [3, 4]. Initial demonstrations based on these fibers have created interest in the scientific community, and efforts are now focused on improving the performance in terms of amplifier gain and laser efficiency [3, 5]. However, few challenges need to be addressed to improve the performance of Bi-doped fiber amplifiers and lasers. Among them, one is the unknown Bi-state that contributes to NIR luminescence, which is heavily dependent on the conditions such as temperature, atmosphere etc. during preform and fiber fabrication [6-8]. Another one is the unwanted losses of these fibers caused by unsaturable loss (UL) and excited state absorption (ESA) [9, 10]. In this paper, we will review the fabrication of Bi-doped aluminosilicate fibers (BASF) and phosphosilicate fibers (BPSF) for $1180 \mathrm{~nm}$ and $1330 \mathrm{~nm}$ wavelength bands, respectively. The $1180 \mathrm{~nm}$ wavelength has specific application in astronomy as a laser guide star and the $1330 \mathrm{~nm}$ wavelength region is to access the O-band for optical fiber communication. The fabricated fibers are characterized for absorption and UL. These measurements are used to optimize the pump wavelength in order to develop efficient Bi-doped fiber amplifiers and lasers.

\section{Results and discussion}
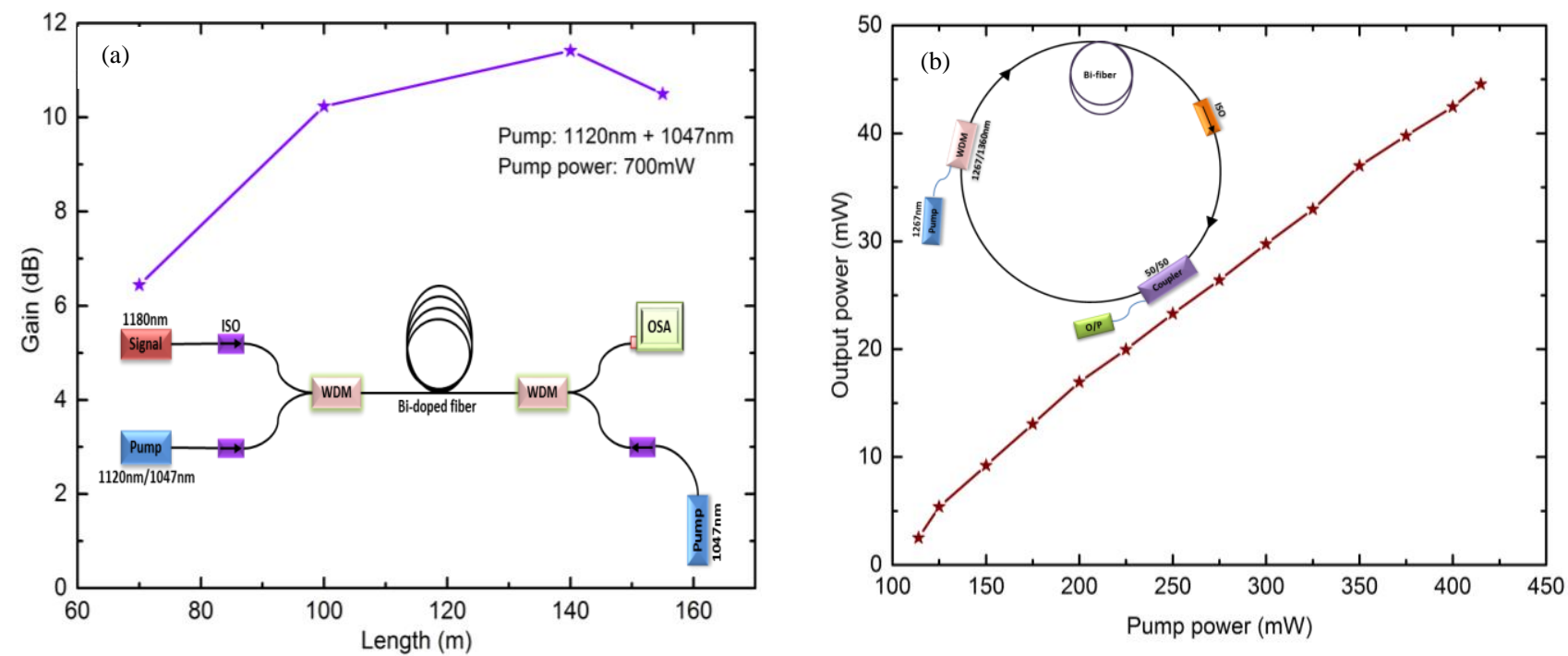

Fig. 1 (a) Gain Vs fiber length in BASF amplifier operating at 1180nm for bi- directional pumping (b) Output power in BPSF laser with launched pump power for 50\% output coupling (OC) with 50m long fiber (Pump and laser wavelengths: 1267 and 1360nm) 
The Bi-doped fiber preforms were fabricated by the modified chemical vapor deposition (MCVD)-solution doping technique. The resultant fibers have the core diameter of 8 and $13 \mu \mathrm{m}$, with an index difference $(\Delta \mathrm{n})$ of 0.008 and 0.004 , for BASF and BPSF, respectively. The cladding diameter of these fibers was $100 \mu \mathrm{m}[11,12]$. The pump wavelength was chosen by measuring the absorption spectra and UL. In BASF, the absorption was 0.35 and $0.7 \mathrm{~dB} / \mathrm{m}$, whereas UL was found to be $35 \%$ and $65 \%$ at 1120 and $1047 \mathrm{~nm}$ pump wavelengths, respectively. The availability of a commercial laser diode (LD) operating at $1120 \mathrm{~nm}$ and low UL measured in BASF made us to explore 1120nm pumping of BASF and compare its performance with more traditional pump wavelength around $1047 \mathrm{~nm}$. The experimental schematic and the corresponding gain characteristics with varying fiber length of a BASF amplifier using bi-directional pumping are shown in Fig.1 (a). Initially, an 1120nm LD with an output power of $350 \mathrm{~mW}$ was used. We obtained a maximum gain of $8 \mathrm{~dB}$ at $1180 \mathrm{~nm}$ for a fiber length of $100 \mathrm{~m}$. The input signal power was $-4 \mathrm{dBm}$. It was observed that $1120 \mathrm{~nm}$ pump provided a gain enhancement of $70 \%$ compared to $1047 \mathrm{~nm}$ pumping. A maximum gain of $11.5 \mathrm{~dB}$ was obtained via simultaneous pumping of BASF at 1120 and $1047 \mathrm{~nm}$ as shown in Fig.1 (a). A relatively small gain enhancement under dual pumping conditions can be explained by an increase in UL with the addition of $1047 \mathrm{~nm}$ pump [11]. This clearly indicates that higher pump power at a wavelength around $1120 \mathrm{~nm}$ will be more suitable to increase gain in BASF operating at $1180 \mathrm{~nm}$. In case of phosphosilicate glass host, three BPSFs were fabricated with different Helium (He)/Oxygen $\left(\mathrm{O}_{2}\right)$ flow ratio during the preform fabrication, while maintaining the total gas flow constant (see Table 1). All other fabrication conditions remain same for all preforms. The preforms were then drawn into fibers (from here onwards named as BPSF-1, BPSF-2, and BPSF-3), respectively. The core and cladding diameters and index difference $(\Delta \mathrm{n})$ between the core and cladding for all BPSFs were same as mentioned at the beginning of this section. The absorption values at 1210 and $1267 \mathrm{~nm}$ pump wavelengths measured by the conventional cut-back method using a white light source are shown in Table 1. We also performed UL measurements in all BPSFs using LDs pumped at 1210 and $1267 \mathrm{~nm}$ wavelengths, respectively. The influence of fabrication conditions on the UL can be seen from Table 1. Due to the low UL and highest lasing performance of BPSF-3 amongst all three BPSFs, the same fiber preform was over jacketed and drawn into a single mode fiber. The cut-off wavelength of the fiber was measured to be around $1100 \mathrm{~nm}$. This single mode BPSF-3 was then used to demonstrate a Bi-doped fiber laser and a wideband amplifier $[12,13]$. A $1267 \mathrm{~nm}$ LD pumped all-fiber laser in a ring cavity has been developed as shown in Fig.1 (b). The absorption at $1267 \mathrm{~nm}$ pump wavelength was found to be $1 \mathrm{~dB} / \mathrm{m}$. The ULs measured for 1267 and $1210 \mathrm{~nm}$ pump wavelengths were $7 \%$ and $14 \%$, respectively. Because of its lower UL at $1267 \mathrm{~nm}$ in BPSF-3, the 1267nm pumping wavelength was chosen from among the available LDs. The laser efficiency was around $14 \%$ for an output coupling ratio of 50/50 with a fiber length of 50m. An output power of $45 \mathrm{~mW}$ at $1360 \mathrm{~nm}$ was obtained as shown in Fig.1 (b).

Table 1. Absorption and UL of BPSFs at two different pump wavelengths

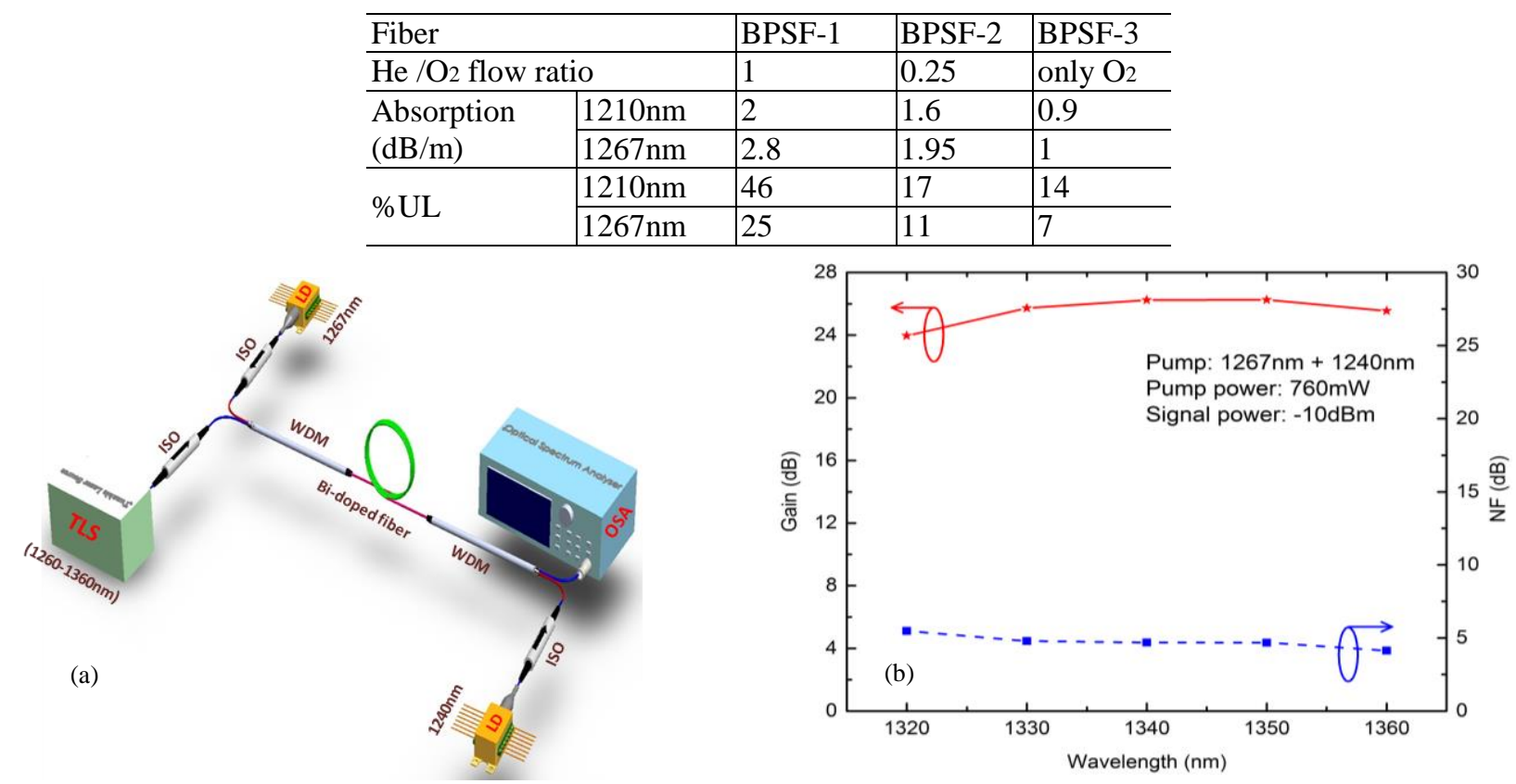

Fig.2 Bi-doped fiber amplifier: (a) Schematic experimental setup, and (b) Gain characteristics with a flat gain of 25 $\pm 1 \mathrm{~dB}$ from 1320-1360nm 
In addition to the aforementioned demonstrations, a Bi-doped phosphosilicate amplifier was constructed as shown in Fig. 2 (a). Initially, the Bi-doped fiber was individually pumped by 1267 and $1240 \mathrm{~nm}$ LDs with pump powers of 360 and $400 \mathrm{~mW}$, respectively to evaluate the gain and noise figure (NF). The input signal power was $-10 \mathrm{dBm}$. The $1267 \mathrm{~nm}$ pumping provided a maximum gain of $15 \mathrm{~dB}$ and a NF of $5 \mathrm{~dB}$ at a wavelength of $1350 \mathrm{~nm}$ for $100 \mathrm{~m}$ long fiber while the gain at $1300 \mathrm{~nm}$ was only $5 \mathrm{~dB}$. In the case of $1240 \mathrm{~nm}$ pump, a maximum gain of $14 \mathrm{~dB}$ and a NF of $6 \mathrm{~dB}$ were obtained at $1330 \mathrm{~nm}$ for $75 \mathrm{~m}$ long fiber. Here, the gain at $1300 \mathrm{~nm}$ was $10 \mathrm{~dB}$, which is double the gain of the $1267 \mathrm{~nm}$ pumping. Thus the $1267 \mathrm{~nm}$ pump can shift the gain toward the longer wavelength whereas the $1240 \mathrm{~nm}$ pump allows the high gain at a shorter wavelength. In order to obtain a flat gain, the Bi-doped fiber was pumped by using both diodes (1267 and 1240nm) simultaneously. The total pump power of the LDs amounted to $760 \mathrm{~mW}$. A flat gain of $25 \pm 1 \mathrm{~dB}$ with a NF of $<6 \mathrm{~dB}$ was achieved over a $40 \mathrm{~nm}$ bandwidth from 1320-1360nm (limited by our tunable laser source, TLS, wavelength range of operation), for the $150 \mathrm{~m}$ long single mode BPSF-3 as shown in Fig. 2 (b). Furthermore, a maximum gain of $29 \mathrm{~dB}$ and a NF of $4.5 \mathrm{~dB}$ were obtained at $1340 \mathrm{~nm}$ for an input signal power of $-30 \mathrm{dBm}$, which, to the best of our knowledge, is the maximum gain, reported from a BDFA operating in the second telecommunication window [13].

\section{Conclusion}

In conclusion, the fabrication conditions and selection of pump wavelengths are critical in order to develop efficient Bi-doped fiber amplifiers and lasers. Also, the present work leads to further investigations on Bi-doped fiber technology to obtain the similar amplifier and laser performance currently offered by the RE-doped fibers. Besides power scaling of Bi-doped fiber lasers is an interesting aspect which requires significant increase of Bi concentration in fiber, suitable for cladding pumping. Research leading to better understanding of the Bi active centers in BASFs and BPSFs would greatly assist further optimization of fiber fabrication.

\section{Acknowledgement}

This work was supported by the UK Engineering and Physical Sciences Research Council grant EP/I01196X/1(Hyperhighway).

\section{References}

[1] D. J. Richardson et. al., "High power fiber lasers: current status and future perspectives [Invited],” J. Opt. Soc. Am. B 27, B63-B92 (2010).

[2] J. He et. al., "Intense ultraviolet photoluminescence at $314 \mathrm{~nm}$ in Gd3+-doped Silica," in Conference on Lasers and Electro-Optics, OSA Technical Digest (online) (Optical Society of America, 2016), paper JTh2A.86.

[3] A. Bufetov et. al., "Bi-doped optical fibers and fiber lasers,” IEEE J. Sel. Top. Quantum Electron. 20, 111-125 (2014).

[4] S. Firstov et. al., "Bismuth-doped optical fibers and fiber lasers for a spectral region of 1600-1800nm,” Opt. Lett. 39, 6927-6930 (2014).

[5] E. M Dianov et. al., "CW bismuth fibre laser,” Quant. Electron. 35, 1083-1084 (2005).

[6] E. Dianov, "Fiber for Fiber Lasers: Bismuth-doped optical fibers: Advances in an active laser media," http://www.laserfocusworld.com/articles/print/volume-51/issue-09.html.

[7] S. Jain et. al., "Thermally-stimulated emission analysis of Bismuth-doped Silica fibers," Opt. Mater. Express, 4, 1361-1366, (2014).

[8] S. Torrengo et. al., "EXAFS studies of the local structures of bismuth centers in multicomponent silica glass based optical fiber preforms," J. Non-Cryst. Solids, 410, 82-87, (2015).

[9] M. P. Kalita et. al., "Bismuth doped fiber laser and study of unsaturable loss and pump induced absorption in laser performance," Opt. Express 16, 21032-21038, (2008).

[10] S. Yoo et. al., "Excited state absorption measurement in the 900-1250nm wavelength range for bismuth-doped silicate fibers," Opt. Lett. 34, 530-532, (2009)

[11] N. K. Thipparapu et. al., "1120 nm diode-pumped Bi-doped fiber amplifier,” Opt. Lett. 40, 2441-2444, (2015).

[12] N. K. Thipparapu et. al., "Diode Pumped Bi-doped Fiber Laser Operating at 1360nm," in Workshop on Specialty Optical Fibers and Their Applications, OSA Technical Digest (online) (Optical Society of America, 2015), paper WT1A.5.

[13] N. K. Thipparapu et. al., "Bi-doped fiber amplifier with a flat gain of 25dB operating in the wavelength band 1320-1360nm," Opt. Lett. 41, 1518-1521, (2016). 